\title{
Learning From Errors in Radiology: A Comprehensive Review
}

\author{
Antonio Pinto, MD, PhD, ${ }^{*}$ Ferdinando Caranci, MD, ${ }^{\dagger}$ Luigia Romano, MD, ${ }^{*}$ \\ Gianpaolo Carrafiello, MD, ${ }^{\neq}$Paolo Fonio, MD,,$^{\S}$ and Luca Brunese, MDף
}

\begin{abstract}
An important goal of error analysis is to create processes aimed at reducing or preventing the occurrence of errors and minimizing the degree of harm. The discovery of any errors presents an opportunity to study the types that occur and to examine their sources and develop measures to prevent them from recurring. The development of an effective system for detecting and appropriately managing errors is essential to substantially attenuate their consequences. At this stage, the error analysis process identifies contributing factors to enable the implementation of concrete steps to prevent such errors from occurring in the future. Active and comprehensive management of errors and adverse events requires ongoing surveillance processes. Educational programs, morbidity and mortality meetings, and a comprehensive and respected root cause analysis process are also essential components of this comprehensive approach. To reduce the incidence of errors, health care providers must identify their causes, devise solutions, and measure the success of improvement efforts. Moreover, accurate measurements of the incidence of error, based on clear and consistent definitions, are essential prerequisites for effective action.

Semin Ultrasound CT MRI 33:379-382 @ 2012 Elsevier Inc. All rights reserved.
\end{abstract}

$\mathrm{M}$ edical errors gained widespread attention with the release of the Institute of Medicine's "To Err Is Human" in November of 1999. This release reported that as many as 98,000 people die each year from inpatient medical errors. Putting this into perspective, deaths from medical errors surpassed deaths from breast cancer, motor vehicle accidents, and acquired immune deficiency syndrome. Furthermore, medication errors account for more deaths annually than workplace injuries. ${ }^{1,2}$ Errors are common in medicine. Fortunately, most errors do not result in significant harm. ${ }^{3}$ Unanticipated outcomes are not necessarily the result of medical error. Common causes of medical errors leading to unanticipated outcomes include limited knowledge, insufficient experience, fatigue, and carelessness. ${ }^{4}$ Unanticipated outcomes not associated with medical error are caused by unrealistic, uncorrected expectations; biological variability; and low-

*Department of Radiology, Cardarelli Hospital, Naples, Italy.

†Unit of Neuroradiology, Department of Diagnostic Imaging and Radiotherapy, Federico II University, Naples, Italy.

¥Department of Radiology, University of Insubria, Varese, Italy.

§Institute of Diagnostic and Interventional Radiology, University of Torino, Turin, Italy

qDepartment of Health Science, University of Molise, Campobasso, Italy.

Address reprint requests to Antonio Pinto, MD, PhD, Department of Radiology, Cardarelli Hospital, Via Posillipo 168/D, I-80123 Naples, Italy. E-mail: antopin1968@libero.it probability, low-risk side effects. Those that are associated with medical error are caused by limited knowledge, inadequate experience, carelessness, and fatigue or faulty medical judgment.

The issue of medical errors is highly complex. To improve health care delivery, we must listen for the alarms of medical errors of all types, and rather than deflecting blame for not hearing them, we must acknowledge our mistakes and learn from them, so that tomorrow when they sound, we are ready and able to respond. Within radiology, the important progress in demonstrating disease has left error analysis a subject infrequently explored. There is the need to analyze the extent and causes of the phenomenon, which would help to identify the most effective measures in terms of clinical risk management, improving education in diagnostic radiology. If we are successful in doing so, we will have made a truly profound improvement in the quality of the health care we deliver.

\section{The Sources of Errors}

Diagnostic errors are relatively common according to Brenner et al. ${ }^{5}$ They may result in delayed diagnosis and treatment, the failure to recognize a complication of treatment, the performance of a study when not indicated or when contraindicated, or the failure to supervise or monitor a case. $^{5}$ 
In general, there are 4 main reasons why radiologists are sued: observer errors, errors in interpretation, failure to suggest the next appropriate procedure, and failure to communicate in a timely and clinically appropriate manner. ${ }^{6}$

Kundel et $\mathrm{al}^{7}$ described 3 types of observer error: scanning error (result of failure of the radiologist to fixate in the area of the lesion), recognition error, and decision-making error, which is the most common error in the study by Kundel et al. ${ }^{7}$ This error is due to incorrect interpretation of a malignant lesion as a normal structure after detection. Another form of observer error that may contribute to lesions being overlooked (a lung cancer, for example) is satisfaction-of-search error. ${ }^{8}$ Satisfaction of search occurs when an abnormality is missed because another abnormality has been detected. Moreover, failures of abnormality detection in film reading (ie, perceptual errors) are subject to psychophysiological factors of human visual perception. ${ }^{9}$ An additional source of error results from the influence a radiology report has over another radiologist. This type of perceptual error occurs because the radiologist reads the old report before looking at the films. ${ }^{10}$ Sources of error in interpretation include clinical history, the presence or absence of previous studies, index of suspicion, the presence of an abnormality, the reading room environment, and the level of vigilance of the interpreter. ${ }^{11}$

Because radiology practice typically involves pivotal interactions with referring physicians regarding diagnoses and care decisions, deficiencies in communication can be devastating.

Even if a radiologist correctly interprets a case, there may be reporting errors. Sources of reporting errors are failure to generate a written report after an examination or a procedure; lack of timeliness; vagueness or ambiguity; subjective probability estimates or verbal expressions of probability, such as "no evidence of;" omission of the degree of certainty regarding findings; recording the wrong patient name; removal of the films from radiology before interpretation; and nonreporting of urgent, significant unexpected, or questionable findings that may not be associated with clinical signs or symptoms. ${ }^{12}$

\section{The Importance of a Good Communication}

In the event of an adverse outcome, patients have clearly voiced their needs and wants, which are as follows: (1) to know the truth about the event and occurrence, (2) for health care organizations to accept responsibility, (3) an apology in recognizing patient trauma, and (4) an apology from the health care practitioner. Monetary reimbursement was not one of the top desires. The need for monetary compensation is exceeded by the patient's desire for human interaction and communication. ${ }^{13}$ Not only is the provision or lack of communication a key factor in malpractice litigation, but the lack of physician communication with disclosure of adverse events is disparaging to patients.

Although a perceived barrier to disclosure is the fear of increased litigation, poor communication is actually a greater risk for litigation. Improved communication can minimize malpractice suits and decrease perceived adverse events. Poor interprofessional communication is a key contributor to adverse events. Poor patient-provider communication, even without an adverse event, may leave patients with a perception of a medical error. These miscommunications, even when no adverse event occurred, also led to obvious patient dissatisfaction and the threat of litigation. ${ }^{14}$ Communication is the key to preventing dissatisfaction, preventing perceived medical error/adverse outcomes, and dealing with adverse outcomes. Although disclosure may be therapeutic for a physician, emotional distress involved with medical errors/adverse outcomes may cause physicians to experience shame and disgrace. Patient dignity must always be respected. Disclosure conversations should include empathy and acknowledgment of what patients and their families have experienced. ${ }^{15}$ Although a disclosure conversation does not imply fault or liability, patients deserve empathy, which may include the expression of "I'm sorry." A lack of consensus exists about the concept of apology, and "I'm sorry" is even more intensely debated. Patients desire an apology for medical errors. If they do not receive an apology, they may perceive the physician as cold and impersonal. Physicians are often reluctant to apologize because they feel it is an admission of guilt and have a fear of increased litigation. ${ }^{16}$

\section{Assessment of Accuracy}

Diagnostic accuracy is perhaps the single most important aspect to monitor in radiology because of its strong connections with health outcomes. At the threshold of uncertainty, radiologists can err on the side of making false-positive or false-negative decisions depending on their concern for the consequences of their decisions. ${ }^{17}$ Which quality assessment approaches are used depends for the most part on the particular aspects of care being examined. Evaluation programs may be person centered or system centered, and their scope can be department wide, institution wide, or system wide. Professional audits and peer reviews, surveys, inspections, and risk management programs are the most common frameworks.

\section{Radiology Peer Review as a Good Way to Reduce Errors}

Peer review has been a component of radiology for many years and has taken on various forms as it has evolved. However, the basic tenet is unchanged-peer review involves oversight among colleagues as a means of ensuring quality care for patients. ${ }^{18}$ In a common model, random studies are pulled from the previous month and given to randomly assigned colleagues for reinterpretation. The original interpretations are correlated with the reinterpretations, and discrepancies are recorded and may be reported to the original radiologists, providing an opportunity for learning and performance improvement. This approach requires the time of the reviewing radiologists and the department personnel 
who pull the studies and record and report results. Peer review has been automated in many radiological departments. Studies are pulled randomly and distributed to reviewers electronically. The reviewers fill out electronic forms, and the results are recorded and reported by e-mail to the original radiologists. ${ }^{18}$

Some departments have integrated peer review into their daily clinical workflow by providing previous interpretations with every new study and including a checkbox for interpreting radiologists to indicate whether they agree with the previous interpretations and, if not, a text box to indicate why they disagree. Discrepancies are reported to the original radiologists by e-mail. This system minimizes the time required of the reviewing radiologists. ${ }^{18}$

When reviewing previous studies to interpret new cases or consult with clinicians, errors may be discovered on the basis of discrepancies in interpretation of the images themselves or additional or revised clinical information. New clinical information, such as operative or pathology results, becomes available that, in retrospect, alters the optimal interpretation of studies; such information may come to light during informal consultations or interdisciplinary conferences..$^{18}$

Radiologists have been encouraged to hold and participate in meetings where cases involving radiological errors are discussed. There is evidence that error rates can reduce after establishment of a departmental discrepancy review meeting, ${ }^{19}$ and such activity can be cited in audit and appraisal discussion.

\section{Educational and Professional Initiatives}

Medical education and training play key roles in ensuring that patients receive the best quality care. The content and methods of teaching and acquiring professional knowledge and skills continually advance in response to developments in science and society. The current major emphasis on improving patient safety and the overall quality of health services has significant implications for medical education.

Federal and state governments, state boards, professional associations, consortiums of institutions, health care organizations, and providers themselves participate in the development and implementation of policies, standards, and guidelines for practice aimed at quality assurance. Improvements in performance have come about through the use of tools such as checklists, clinical history prompts, and perceptual feedback and, more recently, with technologies such as digitally acquired images and computer-aided detection. Double reading, making available previous films and relevant clinical data, careful attention to viewing conditions, and fostering teamwork can also be helpful. ${ }^{20-22}$

New technology can be used to prevent errors. Natural language processing and voice recognition software can be trained to detect errors or discrepancies within reports automatically, before the report is verified or signed off by radiologists. This may not be feasible in developing countries, where digital imaging and voice recognition software are not widely available. In these circumstances, vigilance on part of the reporting radiologist and requesting physician is of importance, to prevent errors in patient treatment. However, even though technological advances, such as structured reporting, electronic requesting (order communication), and voice recognition software, can potentially reduce error in radiology departments, they must be used with caution. Voice recognition software has been shown to increase the error rate in radiology reports as against conventional dictation systems and transcriptionists. Thus, even though technology may reduce a specific type of error in reports, this may occur at the expense of causing another type of error. ${ }^{23}$

Education and leadership will be most important to creating and sustaining a strong safety culture and, arguably, the most important defense against preventable harms.

\section{Organizational Culture of Safety}

Virtually, all the progress in safety thus far has been derived from using multiple converging techniques to discover underlying vulnerabilities and potential paths to failure and innovating ways to cope with the potential form of failure in the context of the changing pressures and demands that is health care. The study of "errorology," the search for the number of errors, is misguided and leads to an unproductive and, ultimately, divisive debate about an inexact, socially charged, and poorly defined quantity. The error or mishap should be the starting point of study, not the ending point. Using error as a cause of break in human performance is wrong because it misses the confounding factors and systems' factors that influence the actual human cognition and performance. The Institute of Medicine report identifies a need to create cultures of safety within all health care organizations. ${ }^{1}$ The health care organization must be a learning environment to build and maintain a culture of safety. Error reporting should be confidential and exist without fear of blame. Reporting should include near misses. Learning from errors and near misses prevents history from repeating itself in potentially devastating ways.

Greater openness with patients about harmful errors is recommended. Many ethicists and professional organizations endorse disclosure of harmful errors to patients..$^{24-27}$

In the United States, the Joint Commission's accreditation standards now require that patients be informed about unanticipated outcomes. ${ }^{28}$ In response, many hospitals are developing disclosure programs. However, recent studies suggest that disclosure of harmful medical errors to patients is the exception rather than the rule. ${ }^{29-34}$ Although disclosing errors is difficult for any physician, radiologists face unique disclosure challenges, especially those who interpret mammograms. ${ }^{35}$

Communicating effectively with patients following errors could enhance patient satisfaction and trust in future health care encounters. ${ }^{36,37}$ Although it may seem counterintuitive, effective disclosure may also reduce the likelihood of malpractice claims. ${ }^{38,39}$ 


\section{Conclusions}

The main reason for studying medical errors is to try to prevent them. Identification and reduction of diagnostic error provides a measure of the efficacy of the health care system, as it reduces mortality, morbidity, length of hospital stays, and additional health care costs.

Diagnostic errors can be reduced by improvements both in knowledge and in systems. An important goal of error analysis is to create processes aimed at reducing or preventing the occurrence of errors and minimizing the degree of harm. Radiology is no more prone than other specialties to quality gaps, and the rate of errors is comparable with all other medical errors. Natural variation in anatomy and subjectivity will always lead to some variation in practice, and a residual error rate will always exist, but advances in technology should help cultivate continued quality improvements.

\section{References}

1. Institute of Medicine, Committee on Quality of Health Care in America: To Err is Human: Building a Safer Health System. Washington, DC, National Academies Press, 1999

2. Institute of Medicine, Committee on Quality of Health Care in America: Crossing the Quality Chasm: A New Health System for the 21st Century. Washington, DC, National Academies Press, 2001

3. Wu AW, Cavanaugh TA, McPhee SJ, et al: To tell the truth: Ethical and practical issues in disclosing medical mistakes to patients. J Gen Intern Med 160:2089-2092, 1997

4. Goldberg RM, Kuhn G, Andrew LB, et al: Coping with medical mistakes and errors in judgment. Ann Emerg Med 39:287-292, 2002

5. Brenner RJ, Lucey LL, Smith JJ, et al: Radiology and medical malpractice claims: A report on the practice standards claims survey of the physician insurers association of America and the American College of Radiology. Am J Roentgenol 171:19-22, 1998

6. Pinto A, Brunese L: Spectrum of diagnostic errors in radiology. World J Radiol 2:377-383, 2010

7. Kundel HL, Nodine CF, Carmody D: Visual scanning, pattern recognition and decision making in pulmonary nodule detection. Invest Radiol 13:175-181, 1978

8. Samuel S, Kundel HL, Nodine CF, et al: Mechanism of satisfaction of search: Eye position recordings in the reading of chest radiographs. Radiology 194:895-902, 1995

9. Yerushalmy J: The statistical assessment of the variability in observer perception and description of roentgenographic pulmonary shadows. Radiol Clin North Am 7:381-393, 1969

10. Berlin L: Malpractice issues in radiology. Alliterative errors. Am J Roentgenol 174:925-931, 2000

11. Pinto A, Acampora C, Pinto F, et al: Learning from diagnostic errors: A good way to improve education in radiology. Eur J Radiol 78:372-376, 2011

12. Physician Insurers Association of America and the American College of Radiology. Practice Standards Claims Survey. Rockville, MD, Physician Insurers Association of America and American College of Radiology, 1997

13. Vincent C, Young M, Phillips A: Why do people sue doctors? A study of patients and relatives taking legal action. Lancet 343:609-613, 1994

14. White AA, Pichert JW, Bledsoe SH, et al: Cause and effect analysis in obstetrics and gynecology. Obstet Gynecol 105:1031-1038, 2005
15. Weiss PM, Miranda F: Transparency, apology and disclosure of adverse outcomes. Obstet Gynecol Clin North Am 35:53-62, 2008

16. Cleopas A, Villaveces A, Charvet A, et al: Patient assessment of a hypothetical medical error: Effects of health outcome, disclosure and staff responsiveness. Qual Saf Health Care 15:136-141, 2006

17. Potchen EJ, Cooper TG, Sierra AE, et al: Measuring performance in chest radiography. Radiology 217:456-459, 2000

18. Halsted MJ: Radiology peer review as an opportunity to reduce errors and improve patient care. J Am Coll Radiol 1:984-987, 2004

19. Espinosa JA, Nolan TW: Reducing errors made by emergency physicians in interpreting radiographs: Longitudinal study. BMJ 320:737740, 2000

20. Robinson PJ: Radiology's Achilles' heel: Error and variation in the interpretation of the roentgen image. Br J Radiol 70:1085-1098, 1997

21. Fitzgerald R: Error in radiology. Clin Radiol 56:938-946, 2001

22. Eklund GW, Cardenosa G, Parsons W: Assessing adequacy of mammographic image quality. Radiology 190:297-307, 1994

23. Sangwaiya MJ, Kalra MK, Roddie M: Venienti occurrite morbo: Knowledge and prevention of errors in radiology. Int J Clin Pract Suppl 65:1-2, 2011

24. Gallagher TH, Studdert D, Levinson W: Disclosing harmful medical errors to patients. N Engl J Med 356:2713-2719, 2007

25. Lo B: Resolving Ethical Dilemmas: A Guide for Clinicians. Philadelphia, PA, Lippincott Williams \& Wilkins, 2005

26. American Society for Health Care Risk Management: Disclosure: What Works Now and What Can Work Even Better. Chicago, IL, American Society for Health Care Risk Management, 2004

27. American Medical Association Council on Ethical and Judicial Affairs: Code of Medical Ethics, Annotated Current Opinions 2004-2005. Chicago, IL, American Medical Association Council on Ethical and Judicial Affairs, 2005

28. The Joint Commission: Hospital Accreditation Standards. Oakbrook Terrace, IL, Joint Commission Resources, 2007

29. Blendon RJ, DesRoches CM, Brodie M, et al: Views of practicing physicians and the public on medical errors. N Engl J Med 347:1933-1940, 2002

30. Studdert DM, Mello MM, Brennan TA: Medical malpractice. N Engl J Med 350:283-292, 2004

31. Gallagher TH, Garbutt JM, Waterman AD, et al: Choosing your words carefully: How physicians would disclose harmful medical errors to patients. Arch Intern Med 166:1585-1593, 2006

32. Hobgood C, Xie J, Weiner B, et al: Error identification, disclosure, and reporting: Practice patterns of three emergency medicine provider types. Acad Emerg Med 11:196-199, 2004

33. Kaldjian LC, Jones EW, Wu BJ, et al: Disclosing medical errors to patients: Attitudes and practices of physicians and trainees. J Gen Intern Med 22:988-996, 2007

34. Wu AW, Folkman S, McPhee SJ, et al: Do house officers learn from their mistakes? JAMA 265:2089-2094, 1991

35. Berlin L: Breast cancer, mammography, and malpractice litigation: The controversies continue. Am J Roentgenol 180:1229-1237, 2003

36. Gallagher TH, Waterman AD, Ebers AG, et al: Patients' and physicians' attitudes regarding the disclosure of medical errors. JAMA 289:10011007,2003

37. Mazor KM, Simon SR, Yood RA, et al: Health plan members' views about disclosure of medical errors. Ann Intern Med 140:409-418, 2004

38. Berlin L: Will saying "I'm sorry" prevent a malpractice lawsuit? Am J Roentgenol 187:10-15, 2006

39. Kachalia A, Shojania KG, Hofer TP, et al: Does full disclosure of medical errors affect malpractice liability? The jury is still out. Jt Comm J Qual Saf 29:503-511, 2003 